\title{
Percepción de autoestima en personas mayores que realizan o no actividad física-deportiva
}

\section{Perceived self-esteem in older people in relation to sports and physical activity}

\author{
Alfonso Javier García y Yolanda Troyano \\ Universidad de Sevilla, España \\ Disponible online 31 de agosto de 2013
}

\begin{abstract}
La autoestima se considera un componente psicológico fundamental de la calidad de vida y del bienestar personal e incluso ha sido propuesta como un área de intervención para mejorar la calidad de vida en la población mayor. El objetivo de este trabajo ha sido analizar la autoestima en personas mayores de 65 años que realizan o no actividad física-deportiva. La muestra la componen 184 personas, de las que 92 realizan actividad física-deportiva y otras 92 no realizan actividad física-deportiva. Mediante una batería de pruebas se recogieron datos sociodemográficos, de frecuencia y duración de la actividad física-deportiva y de autoestima. Los resultados muestran un mayor nivel de autoestima entre las personas mayores que realizan actividad física-deportiva que las que no la realizan. Otros estudios muestran la relación positiva entre la autoestima y la realización de actividad física sobre la base de un programa con actividades y metodologías de carácter colectivo.
\end{abstract}

Palabras clave: Autoestima; Actividad Física-deportiva; Personas Mayores.

Self-esteem is considered a key psychological component of quality of life and personal well-being and has even been proposed as an area of intervention to improve the quality of life in the elderly population. The aim of this study was to analyze self-esteem in people over 65 years old who engaged in sports and physical activities or not. The sample consisted of 184 people; 92 engaged in physical activity and sports and 92 did not. We applied a battery of questionnaires to determine socio-demographic characteristics, the frequency and duration of physical activity and sports, and level of self-esteem. The results show that older people who engage in physical activity and sports have a higher level of self-esteem than those who do not. Based on a program of activities and collective methodologies, other studies have shown a positive relationship between self-esteem and physical activity.

Key words: Self-esteem; Physical Activity and Sports, Older People.

Correspondencia: Alfonso Javier García, Facultad de Psicología, Universidad de Sevilla

C/ Camilo José Cela, s/n, 41018-Sevilla. E-mail: alfonsoj@us.es; E-mail de la segunda autora: Yolanda Troyano: ytroyano@us.es 
La autoestima ha sido estudiada desde una perspectiva multidimensional (Estévez, Martínez y Musitu, 2006) que incluye elementos personales y sociales (Herrera et al., 2004; Martín, Martínez y Martín, 2007). En este sentido, se distingue entre "Autoestima Personal", referida a la evaluación positiva o negativa de los atributos personales tanto privados como interpersonales; y "Autoestima Colectiva", entendida como el grado en que una persona es consciente de evaluar su pertenencia a grupos o categorías sociales (Sánchez, 1999) y destaca la importancia de la identidad grupal (Gonsalkorale, Carlisle y Von Hippel, 2007; Jiménez, Páez y Javaloy, 2005; Liu y Dresler-Hawke, 2006).

Referente al carácter multidimensional de la autoestima, Rubin y Hewstone (1998) conceptualizan las diferentes dimensiones de la autoestima como rasgo-estado, global-específica y personal-social. La "Autoestima como rasgo" se refiere al producto de autoevaluaciones hechas sobre un periodo relativamente largo de tiempo; mientras que la "Autoestima como estado" es el producto de autoevaluaciones llevadas a cabo en el presente inmediato, y a consecuencia de experiencias vitales concretas. Estudios recientes analizan la autoestima desde la teoría de la Identidad Social (García-Prieto y Scherer, 2006), o la autoestima colectiva (Lam, 2008) entendidas como rasgo o estado. Rosenberg, Schooler, Schoenbach y Rosenberg (1995) exponen que la "Autoestima global" hace referencia a la propia imagen a nivel general, mientras que la "Autoestima específica" se refiere a la autoimagen particular, con relación a un tipo concreto de evento o circunstancia. En relación con estos constructos hay autores que afirman que una adecuada autoestima global permite arriesgarnos a afrontar situaciones novedosas, mientras que, una baja autoestima global dificulta la identificación de recursos maximizando las limitaciones al afrontar cualquier experiencia (Arranz y Coca, 2006). La "Autoestima personal" hace referencia al grado de estima que tiene la persona sobre sí misma. La "Autoestima social” es el grado de estima proporcionado por la autoimagen que deriva de los endogrupos de pertenencia (Luhtanen y Crocker, 1992). Hoy día, esta idea destaca la concepción de que los miembros de los grupos se esfuerzan por conseguir una autoestima positiva como miembros de tales grupos, más que como individuos (Kaiser, Hagiwara, Malahy y Wilkins, 2009).

En lo que se refiere a los distintos modelos teóricos que han considerado la autoestima, la teoría de la Identidad Social ha tenido gran influencia en la Psicología Social contemporánea proporcionando contribuciones a la comprensión de la dimensión social del comportamiento (Ma, 2008; Scandroglio, López y San José, 2008). Desde la "Teoría de la Identidad Social" formulada por Tajfel y Turner (1986) se sugiere que existe una relación entre la autoestima personal y la autoestima colectiva en la medida que ambas forman parte de un concepto más amplio y general que es el autoconcepto. De acuerdo con esta teoría, las personas no sólo organizan y simplifican la complejidad del contexto social que les rodea, sino que además construyen un marco de referencia para su identidad social. Ésta es entendida como aquel componente del sí-mismo que se deriva de la pertenencia a colectivos sociales. La "Teoría de la Identidad Social" postula que las personas están motivadas por mantener y proyectar un sí-mismo coherente y positivo, y esto lo logran a través de los grupos sociales a los que pertenecen. Así, una valoración positiva del endogrupo asegura una valoración positiva del sí-mismo (Pérez y Smith, 2008), lo cual permite la construcción de la identidad social en el contexto de las actividades grupales (Vera, Rodríguez y Grubits, 2009). Una identidad social positiva se relaciona con altos niveles de autoestima colectiva en las relaciones endogrupales (Eliezer, Major y Mendes, 2010; Lewis y Neighbors, 2007; Luhtanen y Crocker, 2005; Scheepers, Spears, Manstead y Doosje, 2009; Ysseldyk, Matheson y Anisman, 2010); mientras que una situación de estrés, ansiedad o depresión afecta al autocontrol y la autoestima (Crocker, Moeller y Burson, 2010).

El autoconcepto y la autoestima aparecen como componentes psicológicos fundamentales de la calidad de vida durante la adultez mayor (Trujillo, 2005) y del bienestar personal (García, Musitu y Veiga, 2006). La autoestima ha sido propuesta como un área de intervención para mejorar la calidad de vida en la población mayor (Rodríguez, Valderrama y Uribe, 2010). Herrera et al. (2004) consideran la autoestima como predictor de la calidad de vida en las personas mayores sobre la base de una alta autoestima familiar y social y una baja autoestima intelectual, física y emocional. La autoestima también se ve reforzada por el apoyo social, de manera que mientras más extensa sea la red de apoyo social, mejor autoestima mantiene la persona mayor (Fernández y Manrique-Abril, 2010). Los cambios socioeconómicos y de salud física asociados a la edad suelen ir asociados a una disminución en la autoestima (Orth, Trzesniewski y Robins, 2010). No obstante, una investigación identifica los aspectos positivos del proceso de envejecimiento y los relaciona con la autonomía y la percepción de salud de los mayores, resultando que el sentimiento de utilidad, la capacidad de tomar decisiones y tener aficiones e ilusiones se asocian a una independencia instrumental y adecuada autoestima (López et al., 2006).

La autoestima ha sido estudiada en relación con la realización de actividades cotidianas (Durán, Orbegoz, UribeRodríguez y Uribe, 2008; Montorio, 2007), así como entre la percepción de autoeficacia, la autoestima y el bienestar psicológico con la capacidad para realizar dichas actividades (Ortíz y Castro, 2009). Algunos autores (Bárcena, Iglesias, Galán y Abella, 2009; Dorantes, Ávila, Mejía y Gutiérrez, 2007) señalan que bajas puntuaciones en autoestima y en bienestar autopercibido se relacionan con la dependencia funcional para realizar actividades básicas e instrumentales de la vida diaria en personas mayores. El deterioro progresivo que implica el envejecimiento ha centrado el interés de estudios que analizan las actitudes que tiene la sociedad sobre las personas mayores y cómo éstas influyen en la imagen que los mayores tienen de 
sí mismos. De manera que éstos comparten una autoimagen de vejez negativa en la que prevalecen conceptos estereotipados como inutilidad, enfermedad, deterioro y carga para los demás (Arrieta y Espinosa, 2005; Shaw, Liang y Krause, 2010; Ulla et al., 2003). La imagen negativa que se origina en la persona mayor se relaciona con lo que Reig (2004) denomina "patrón cerrado a la vida", donde los hechos, los cambios y las dificultades son percibidos como amenazas, injusticias y pérdidas irreparables. En el lado opuesto se sitúa el "patrón abierto a la vida", en el que la persona mayor tiende a percibir los hechos, los cambios y las dificultades de la vida como desafíos a resolver, como situaciones susceptibles de aprendizaje, como ganancias en descubrimiento y comprensión.

Desde una perspectiva positiva, los resultados avalan que el autoconcepto de las personas mayores no sufre disminuciones con la edad, y que continúa siendo un recurso personal adaptativo, flexible, dinámico y resistente muy poderoso y valioso para una adaptación satisfactoria y con éxito en la vejez (Fragoeiro, Pestana y Paul, 2007; Rojas, Toronjo, Rodríguez y Rodríguez, 2006).

Se ha comprobado que los estudios que relacionan el autoconcepto y la autoestima con distintas variables psicosociales han sido muy numerosos, sin embargo, uno de los aspectos más destacados es el relacionado con los aspectos físicos (Guillén y Ramírez, 2011). Estas relaciones han sido bidireccionales: desde aquellas que establecen relaciones entre la influencia del autoconcepto físico sobre la realización de actividades físicas y deportivas (Guérin, Marsh y Famose, 2004; Marsh, Richards, Johnson, Roche y Tremayne, 1994), a las que analizan la influencia de la actividad física y deportiva sobre el autoconcepto y la autoestima (Guillén y Sánchez, 2003; Landers y Arent, 2001; Moreno, Cervelló y Moreno, 2008).

En el ámbito de la actividad física y el deporte se han intentado establecer múltiples relaciones entre las variables implicadas en la dimensión de la autoestima. Así, Moreno, Moreno y Cervelló (2007) relacionaban la intención de ser físicamente activo y el autoconcepto físico; Pedersen y Seidman (2004) relacionaban el tiempo dedicado al ejercicio físico con el grado de autoestima; Alcántara, Ureña y Garcés de los Fayos (2002) evidencian científicamente que el ejercicio físico mejora la intensidad de la sintomatología, la condición física y la calidad de vida en diferentes tipos de población; o Rodríguez, Wigfield y Eccles (2003) que relacionaron el impacto que tienen sobre la autoestima las valoraciones realizadas sobre el deporte o la actividad física.

Las personas mayores que practican actividad físicadeportiva son todavía escasas en comparación con otros grupos sociales debido a la existencia de circunstancias, obstáculos o barreras (Campos, Jiménez-Beatty, González, Martín e Hierro, 2011) aunque algunos autores (Bergland, Thorsen y Loland, 2010; Choia, Jangb, Leea y Kima, 2010) concluyen que gozar de una alta autoestima correlaciona positivamente con la realización de actividad física.
El objetivo de este trabajo ha sido analizar las diferencias en la autoestima personal percibida entre personas mayores que realizan o no actividad física-deportiva. Se considera que las personas que realizan actividad física-deportiva perciben una mejor autoestima personal que aquellas que no la realizan.

\section{Participantes}

Se realizó un muestreo intencional por accesibilidad que permitió localizar las personas participantes en el estudio que fueron seleccionadas por sexo, edad, estado civil y estudios. Participaron un total de 184 personas mayores de 65 años, de las que 92 estaban adscritas a programas de salud y calidad de vida impartidos en centros de día de zonas urbanas y participaban en programas de actividad física-deportiva, y las otras 92 no participan en programas de actividad física-deportiva. El total de los 92 participantes que realizan actividad físicadeportiva lo hacen con una frecuencia de al menos dos sesiones y con una duración mínima de dos horas semanales. La muestra formada por 115 mujeres y 69 hombres con una media de edad de $M=71.3$ años, con una $D T=2.43$. Por grupos de edad, participaron 133 personas con edades comprendidas entre los 65 y 74 años y 51 personas mayores de 75 años. Por estado civil, participaron 104 personas casadas, 43 viudas, 22 solteras, 13 divorciadas y 2 en parejas de hecho. Por estudios, participaron 63 personas con estudios primarios, 60 con estudios secundarios, bachilleres y formación profesional, 38 con diplomaturas y 23 con licenciatura.

\section{Instrumentos}

Se ha empleado una batería de pruebas compuesta por un cuestionario de datos sociodemográficos y de implicación en actividad física-deportiva diseñado ad hoc y un cuestionario estandarizado en su versión española (Escala de Autoestima de Rosenberg, 1965). El cuestionario, en su parte de datos sociodemográficos consta de cuatro ítems (sexo, edad, estado civil y estudios) y en la de implicación en actividad física-deportiva consta de dos ítems (frecuencia y duración). La escala de autoestima consta de diez preguntas con un formato tipo Likert de cuatro puntos que miden la actitud hacia uno mismo y hacia los demás. La mitad de los ítems están enunciados en positivo (ítems 1, 2, 4, 6 y 7) y la otra mitad en negativo (ítems 3, 5, 8, 9 y 10). La graduación de respuestas tiene 4 puntos ( $1=$ muy en desacuerdo, 2 = en desacuerdo, $3=$ de acuerdo y $4=$ muy de acuerdo) y se asigna el puntaje inverso a las afirmaciones direccionadas negativamente. En estudios realizados con muestras similares la fiabilidad de la Escala de Rosenberg ha sido de 0.79 (Fernández, 2002). Aunque se ha aplicado el instrumento original por adecuarse a los objetivos de investigación, éste se ha sometido a una prueba de validez de contenido, siendo validado por cinco investigadores externos al equipo de trabajo. Todos los participantes comprendieron los ítems y los valores de respuesta asociados. El índice de consistencia interna ha sido de un Alpha de Cronbach de $\alpha=.892$. 


\section{Procedimiento}

Un investigador, entrenado previamente sobre la administración del instrumento, se encargó de entrevistar a personas mayores adscritas y no adscritas a programas de salud y calidad de vida en Centros de Día. Se intentó crear en todo momento un clima de confianza durante la entrevista, haciendo referencia a los objetivos de trabajo, al carácter confidencial de las respuestas, insistiendo que no había respuestas correctas ni incorrectas, y que lo que importaba era su opinión y sinceridad respecto a lo preguntado y responder a todas las preguntas. Todos los participantes, realizaran o no actividad física-deportiva, dieron su consentimiento por escrito.

Tras registrar las respuestas de las personas entrevistadas, se codificaron y analizaron los datos con el paquete estadístico SPSS 14.0.

\section{Resultados}

Se ha dividido la muestra en dos grupos. El primer grupo lo componen las 92 personas que realizaban dos sesiones de actividad física-deportiva durante al menos dos horas a la semana de forma regular y, el segundo grupo, las 92 personas que no realizaban actividad física-deportiva.

Con el fin de contrastar el grupo que realiza actividad físicadeportiva con el grupo que no realiza actividad física-deportiva se comprueba en primer lugar el supuesto de normalidad de las variables. Los valores del estadístico Kolmogorov-Smirnov muestran que el grado de significación mayor que el nivel $\alpha=$ .05 nos hace aceptar la hipótesis de normalidad de distribución de los datos.

Las personas que realizan dos sesiones de actividad físicodeportiva con una dedicación mínima de dos horas semanales de forma regular presentan un mayor nivel de autoestima $(M=$ $36.1 ; D T=1.1)$ que aquellas que no realizan actividad físicadeportiva $(M=26.3 ; D T=1.4)$. Para conocer si esta diferencia de medias es significativa a un nivel de $\alpha=.05$ se realiza la prueba de Levene para contrastar si la varianza cumple el supuesto de homogeneidad de varianzas entre ambos grupos. La prueba indica que se cumple el supuesto de homogeneidad de la varianza $p=.176(F=1.949, p>.05)$. Los resultados muestran que la diferencia de medias es estadísticamente significativa $\left(\mathrm{t}_{96}=3.11 ; p<.05\right)$. Además, el resultado del tamaño del efecto de la realización de la actividad física-deportiva en el nivel de autoestima es elevado $(r=.66)$.

\section{Discusión}

Los resultados obtenidos en el presente trabajo permiten plantear que las personas que realizan dos sesiones de actividad física-deportiva durante al menos dos horas a la semana de forma regular presentan un mayor nivel de autoestima que aquellas que no realizan actividad física-deportiva.

La persona mayor que realiza actividades físicas con frecuencia muestra un patrón abierto a la vida a la hora de afrontar la vejez, creyéndose valiosa y capaz, sintiéndose bien consigo misma y con los demás (Reig, 2004) y, las que se perciben con actitudes físicas favorables tienen un mejor autoconcepto que el resto (Sonstroem, 1998). Igualmente, una persona que percibe su habilidad como alta, mantendrá una actitud más positiva hacia esa actividad y se sentirá más motivada a realizarla (Piéron, 1998).

Asimismo, Rojas, Toronjo, Rodríguez y Rodríguez (2006) concluyen que para potenciar la autonomía de la persona mayor, redundante en una mayor autoestima, es necesario que las instituciones de las que forman parte se adapten a las necesidades verdaderas de aquellas, adaptando la organización institucional si fuera necesario. Esto podría contextualizarse desde uno de los postulados de la teoría de la Identidad Social, según el cual las personas están motivadas por mantener y proyectar un sí-mismo coherente y positivo, que lo consiguen a través de los grupos sociales a los que pertenecen (Pérez y Smith, 2008).

Esta investigación no está exenta de limitaciones. Por un lado, sería interesante utilizar, además del Cuestionario Rosenberg empleado en el ámbito de la Psicología del Deporte y en otras áreas como la Medicina y la Psicología (Bektas y Ozturk, 2008; Mouttapa, Weiss y Hermann, 2009), instrumentos como el de Luhtanen y Crocker (1992) que evalúa la dimensión colectiva del constructo, y así profundizar en el análisis del carácter grupal que tiene la realización de cualquier actividad física. Por otro lado, es importante considerar que la mayoría de los trabajos sobre autoestima y actividad física se ha realizado con población joven o adolescente (Asci, 2003) y debido al aumento de la curva demográfica en el sector correspondiente a la población mayor sería muy interesante incrementar el número de estudios en este grupo de edad.

En conclusión, la realización de actividad física-deportiva por personas mayores influye positivamente sobre su percepción de autoestima personal. No obstante, habrá que tener en cuenta que la influencia de la práctica de actividad física-deportiva sobre la autoestima está sobredimensionada como concluyen McAuley et al. $(1997,2000)$ y Spence, McGannon y Poon (2005). En este sentido, es fundamental proponer estudios de carácter longitudinal que permitirían analizar los efectos de la actividad física-deportiva en la autoestima a lo largo del ciclo vital.

\section{Referencias}

1. Alcántara, P., Ureña, F. y Garcés de los Fayos, E.J. (2002). Repercusiones de un programa de actividad física gerontológica sobre la aptitud física, autoestima, depresión y afectividad. Cuadernos de Psicología del Deporte, 2, 57-73.

2. Arranz, P. y Coca, C. (2006). Desarrollo personal. En M.L. Sarrate (Coord.), Atención a las personas mayores. Intervención práctica (pp. 125-157). Madrid: Universitas, S.A.

3. Arrieta, C. y Espinosa, J. (2005). El boom de los abuelitos. Una investigación sobre la imagen que los mayores tienen de si mismos y de la vejez. Recuperado el 4 de abril de 2009 de http://www.enplenitud.com/nota.asp?articuloID=3190. 
4. Asci, F.H. (2003). The effects of physical fitness training on trait anxiety and physical self-concept of female university students. Psychology of Sport and Exercise, 4, 255-264. http://dx.doi.org/10.1016/S1469-0292(02)00009-2

5. Bárcena, C., Iglesias, J.A., Galán, M.I. y Abella, V. (2009). Dependencia y edadismo. Implicaciones para el cuidado. Revista Enfermería CyL, 1, 46-52.

6. Bektas, M. y Ozturk, C. (2008). Effect on health promotion education on presence of positive health behaviors, level of anxiety and self-concept. Social Behavior \& Personality, 36, 681-690. http://dx.doi.org/10.2224/sbp.2008.36.5.681

7. Bergland, A., Thorsen, K. y Loland, N.W. (2010). The relationship between coping, self-esteem and health on outdoor walking ability among older adults in Norway. Ageing \& Society, 30, 949-963. http://dx.doi.org/10.1017/ $\underline{\mathrm{S} 0144686 \mathrm{X} 1000022 \mathrm{X}}$

8. Campos, A., Jiménez-Beatty, J.E., González, M.D., Martín, M. e Hierro, D. (2011). Demanda y percepción del monitor de las personas mayores en la actividad física y deporte en España. Revista de Psicología del Deporte, 20, 61-77. http://dx.doi.org/10.1016/j.archger.2010.08.011

9. Choia, K.B., Jangb, S.H., Leea, M.L. y Kima, K.H. (2010). Sexual life and self-esteem in married elderly. Archives of Gerontology and Geriatrics, 53, 17-20. http://dx.doi. org/10.1016/j.archger.2010.08.011

10. Crocker, J., Moeller, S. y Burson, A. (2010). Implications for Self-Regulation. En R.H. Holey (Ed.), Handbook of Personality and Self-Regulation (pp. 403-446). UK: WileyBlackwell.

11. Dorantes, G., Ávila, J.A., Mejía, S. y Gutiérrez, L.M. (2007). Factores asociados con la dependencia funcional en los adultos mayores: un análisis secundario del Estudio Nacional sobre Salud y Envejecimiento en México, 2001. Revista Panamericana de Salud Publica, 22, 1-11. http:// dx.doi.org/10.1590/S1020-49892007000600001

12. Durán, D., Orbegoz, L.J., Uribe-Rodríguez, A.F. y Uribe, J.M. (2008). Social Integration and Functional Skills in Older Adults. Universitas Psychologica, 7, 263-270.

13. Eliezer, D., Major, B. y Mendes, W.B. (2010). The costs of caring: Gender Identification increases threat following exposure to sexim. Journal of Experimental Social Psychology, 46, 159-165. http://dx.doi.org/10.1016/j. jesp.2009.09.015

14. Estévez, E., Martínez, B. y Musitu, G. (2006). La autoestima en adolescentes agresores y víctimas en la escuela: La perspectiva multidimensional. Intervención Psicosocial, 15, 223-232.

15. Fernández, R. y Manrique-Abril, F.G. (2010). Rol de la enfermería en el apoyo social del adulto mayor. Enfermería Global, 19, 1-9.

16. Fragoeiro, I., Pestana, M.H. y Paul, C. (2007). Mental health of the older people from the self-governing Region of Madeira: Pilot study. Gerokomos, 18, 23-27.
17. García, J.F., Musitu, G. y Veiga, F. (2006). Autoconcepto en adultos de España y Portugal. Psicothema, 18, 551-556.

18. García-Prieto, P. y Scherer, K. (2006). Connecting social identity theory to cognitive appraisal theory of emotions. En R. Brown \& D. Capozza (Eds.), Social identities: Motivational, emotional, cultural influences (pp. 189-207). East Sussex, UK: Psychology Press.

19. Gonsalkorale, K., Carlisle, K. y Von Hippel, W. (2007). Intergroup Threat Increases Implicit Stereotyping. International Journal of Psychology and Psychological Therapy, 7, 189-200.

20. Guérin, F., Marsh, H.W. y Famose, J.P. (2004). Generalizability of the PSDQ and its relationship to physical fitness: The European French Connection. Journal of Sport \& Exercise Psychology, 26, 19-38.

21. Guillén, F. y Ramírez, M. (2011). Relación entre el autoconcepto y la condición física en alumnos de Tercer Ciclo de Primaria. Revista de Psicología del Deporte, 20, 45-59.

22. Guillén, F. y Sánchez, R. (2003). Autoconcepto en jóvenes sedentarios y practicantes deportivos. Edupsykhé, 2, 259272.

23. Herrera, J.M., Barranco, C., Melián, C., Herrera, R.M., Rodríguez, M.I. y Mesa, M.N. (2004). La autoestima como predictor de la calidad de vida en los mayores. Portularia, 4, 171-177.

24. Jiménez, A., Páez, D. y Javaloy, F. (2005). Correlatos psico-sociales de la participación en manifestaciones tras el atentado del 11 de Marzo. Revista de Psicología Social, 20, 263-275. http://dx.doi.org/10.1174/021347405774277686

25. Kaiser, C.R., Hagiwara, N., Malahy, L.W. y Wilkins, C.L. (2009). Group identification moderates attitudes toward ingroup members who confront discrimination. Journal of Experimental Social Psychology, 45, 770-777. http:// dx.doi.org/10.1016/j.jesp.2009.04.027

26. Lam, B. (2008). The Function of Acculturation and Collective Self-Esteem on Prejudicial Attitudes among Vietnamese American Young Adults. Journal of Human Behavior in the Social Environment, 18, 350-363. http://dx.doi. org/10.1080/10911350802427621

27. Landers, D.M. y Arent, S.M. (2001). Physical activity and mental health. En R.N. Singer, H.A. Hausenblas y C.M. Janelle (Eds.), Handbook of sport psychology ( $2^{\mathrm{a}}$ ed., pp. 740-765). Nueva York: John Wiley \& Sons.

28. Lewis, M.A. y Neighbors, C. (2007). Optimizing Personalized Normative Feedback: The Use of Gender-Specific Referents. Journal of Studies on Alcohol and Drugs, 68, 228-237.

29. Liu, J.H. y Dresler-Hawke, E. (2006). Collective shame and the positioning of german national identity. Psicología Política, 32, 130-153.

30. López, R.M., Colas, M.I., Hernández, E., Ruíz, E.M., Padín, C. y Morell, R. (2006). Envejecer: aspectos positivos, capacidad funcional, percepción de salud y síndromes 
geriátricos en una población mayor de 70 años. Enfermería Clínica, 16, 27-34. http://dx.doi.org/10.1016/S11308621(06)71174-3

31. Luhtanen, R.K. y Crocker, J. (1992). A collective selfesteem scale: self-evaluation of one social identity. Personality and Social Psychology Bulletin, 18, 302-318. http:// dx.doi.org/10.1177/0146167292183006

32. Luhtanen, R.K. y Crocker, J. (2005). Alcohol Use in College Students: Effects of Level of Self-Esteem, Narcissism, and Contingencies of Self-Worth. Psychology of Addictive Behaviors, 19, 99-103. http://dx.doi.org/10.1037/0893$\underline{164 X .19 .1 .99}$

33. Ma, L. (2008). Work-based self-esteem: Conceptual, theorical, and scale development and model testing. Dissertation Abstracts International: Section A: Humanities and Social Sciences, 69 (5A), 1875.

34. Madariaga, J.M. y Goñi, A. (2009). El desarrollo psicosocial. Revista de Psicodidáctica, 14, 93-116.

35. Marsh, H.W., Richards, G.E., Johnson, S., Roche, L. y Tremayne, P. (1994). Physical Self-Description Questionnaire: Psychometric properties and a multitrait-multimethod analysis of relations to existing instruments. Journal of Sports \& Exercise Psychology, 16, 270-305.

36. Martín, M.J., Martínez, J.M. y Martín, A. (2007). Psicología Social contemporánea e intervención social. Boletín de Psicología, 91, 55-82.

37. McAuley, E., Blissmer, B., Katula, J., Duncan, T.E. y Mihalko, S.L. (2000). Physical activity, self-esteem and self-efficacy relationships in older adults: A randomized controlled trial. Annals of Behavioral Medicine, 22, 131-139. http:// dx.doi.org/10.1007/BF02895777

38. McAuley, E., Mihalko, S.L. y Bane, S.M. (1997). Exercise and self-esteem in middel-aged adults: Multidimensional relationships and physical-fitness and self-efficacy influences. Journal of Behavioral Medicine, 20, 67-83. http:// dx.doi.org/10.1023/A:1025591214100

39. Montorio, I. (2007). Intervenciones psicológicas en la prevención y atención a la dependencia. Intervención Psicosocial, 16, 43-54.

40. Moreno, J.A., Cervelló, E. y Moreno, R. (2008). Importancia de la práctica físico-deportiva y del sexo en el autoconcepto físico de los 9 a los 23 años. International Journal of Clinical and Health Psychology, 8, 171-183.

41. Moreno, J.A., Moreno, R. y Cervelló, E. (2007). El autoconcepto físico como predictor de la intención de ser físicamente activo. Psicología y Salud, 17, 261-267.

42. Mouttapa, M., Weiss, J.W. y Hermann, M. (2009). Is Image Everything? The Role of Self-Image in the Relationship Between Family Functioning and Substance Use Among Hispanic Adolescents. Substance \& Misuse, 44, 702-721. http://dx.doi.org/10.1080/10826080802486830

43. Orth, U., Trzesniewski, K.H. y Robins, R.W. (2010). SelfEsteem Development From Young Adulthood to Old Age: A Cohort-Sequential Longitudinal Study. Journal of Perso- nality and Social Psychology, 98, 645-658. http://dx.doi. org/10.1037/a0018769

44. Ortíz, J.B. y Castro, M. (2009). The psychological wellbeing of the elderly and their relation with self-esteem and self- efficiency: nursing contribution. Ciencia y Enfermería, 15, 25-31.

45. Pedersen, S. y Seidman, E. (2004). Team Sports achievement and self-esteem development among urban adolescents girls. Psychology of Women Quarterly, 28, 412-422. http://dx.doi.org/10.1111/j.1471-6402.2004.00158.x

46. Pérez, R. y Smith, V. (2008). Comunicación Intergeneracional: El Cuestionario de Percepción de la Comunicación Intergeneracional (CPCI) en el Contexto Costarricense. Interamerican Journal of Psychology, 42, 589603.

47. Piéron, M. (1998). Perspectivas de la Educación Física en el siglo XXI. En J. Hernández, U. Castro y H. Cruz (Eds.), Educación física escolar y deporte de alto rendimiento (pp. 19-45). Las Palmas: ACCAFIDE.

48. Ramos de Oliveira, R. y Páez, D. (2007). Diferencias en auto-estima conforme la edad y etnicidad. Actas del X Congreso Nacional de Psicología Social. Cádiz: Universidad de Cádiz.

49. Reig, A. (2004). Psicología de la vejez. Comportamiento y adaptación. En R. Fernández-Ballesteros (Dir.), Gerontología Social (pp. 167-200). Madrid: Pirámide Psicología.

50. Rodríguez, A.F., Valderrama, L.J. y Uribe, J.M. (2010). Intervención psicológica en adultos mayores. Psicología desde el Caribe, 25, 246-258.

51. Rodríguez, D., Wigfield, A. y Eccles, J.S. (2003). Changing competence perceptions, changing values: Implications for youth sport. Journal of Applied Sport Psychology, 15, 67-81. http://dx.doi.org/10.1080/10413200305403

52. Rojas, M.J., Toronjo, A., Rodríguez, C. y Rodríguez, J.B. (2006). Autonomía y estado de salud percibidos en ancianos institucionalizados. Gerokomos, 17, 8-23.

53. Rosenberg, M. (1965). Society and the adolescent self image. Princenton, N.J.: University Press.

54. Rosenberg, M., Schooler, C., Schoenbach, C. y Rosenberg, F. (1995). Global Self-Esteem and Specific SelfEsteem: Different Concepts, Different Outcomes. American Sociological Review, 60, 141-156. http://dx.doi. org/10.2307/2096350

55. Rubin, M. y Hewstone, M. (1998). Social identity theorys self-esteem hypothesis: a review and some suggestions for clarification. Personality and Social Psychology Review, 2, 40-62. http://dx.doi.org/10.1207/s15327957pspr0201_3

56. Sánchez, E. (1999). Relación entre la autoestima personal, la autoestima colectiva y la participación en la comunidad. Anales de Psicología, 15, 251-260.

57. Scandroglio, B., López, J.S. y San José, M.C. (2008). La Teoría de la Identidad Social: una síntesis crítica de sus fundamentos, evidencias y controversias. Psicothema, 20, 80-89. 
58. Scheepers, D., Spears, R., Manstead, A.S. y Doosje, B. (2009). The influence of discrimination and fairness on collective self-esteem. Personality and Social Psychology Bulletin, 35, 506-515. http://dx.doi. org $/ 10.1177 / 0146167208329855$

59. Shaw, B.A., Liang, J. y Krause, N. (2010). Age and Race Differences in the Trajectories of Self-Esteem, Psychology and Aging, 25, 82-94. http://dx.doi.org/10.1037/a0018242

60. Spence, J.C., McGannon, K.R. y Poon, P. (2005). The Effect of Exercise on Global Self-Esteem: A Quantitative Review. Journal of Sport \& Exercise Psychology, 27, 311-334.

61. Tajfel, H. y Turner, J.C. (1986). An integrative theory of intergroup relations. En S. Worchel y W.G. Austin (Eds.), Psychology of intergroup relations (pp. 7 - 24). Chicago: Nelson-Hall.

62. Trujillo, S. (2005). Agenciamiento individual y condiciones de vida. Universitas Psychologica, 4, 221-229.
63. Ulla, S., Coca, C., Rincón, C., Díaz, J.L., Remor, E., Arranz, P. y Bayés, R. (2003). Coping with Death: Perceptions of health care professionals working in a pediatric intensive care unit and a geriatric service. Illnes, Crises \& Loss, 12. http://dx.doi.org/10.1177/1054137303256534

64. Vera, J.A., Rodríguez, C.K. y Grubits, S. (2009). Social psychology and the concept of culture. Psicologia \& Sociedade, 21, 100-107.

65. Ysseldyk, R., Matheson, K. y Anisman, H. (2010). Religiosity as Identity: Toward an Understanding of Religion From a Social Identity Perspective. Personality and Social Psychology Review, 14, 60-71.

Fecha de recepción: 4 de marzo de 2013 Fecha de recepción de la versión modificada: 31 de mayo de 2013

Fecha de aceptación: 18 de junio de 2013 